\title{
Elementos para abordar la necesidad de información de las familias en una unidad de cuidados intensivos*
}

\author{
Elements for Approaching the Information Needs of the Family at the ICU \\ Elementos para abordar a necessidade de informação das famílias em uma unidade de terapia intensiva
}

\section{Laura Carolina Boada Quijano ${ }^{\text {a }}$ \\ Universidad Industrial de Santander, Colombia \\ lcboadaq@unal.edu.co \\ ORCID: http://orcid.org/0000-0001-6065-0476}

Sandra Rocio Guáqueta Parada

Universidad Nacional de Colombia, Colombia

ORCID: http://orcid.org/0000-0002-2354-7493
DOI: https://doi.org/10.11144/Javeriana.ie21-2.eani

Fecha de recepción: 28 Junio 2018

Fecha de aprobación: 11 Abril 2019

Fecha de publicación: 30 Noviembre 2019

\section{Resumen:}

Introducción: el cuidado del paciente crítico de forma holística debe incluir el abordaje de la familia, que experimenta necesidades específicas derivadas de la situación crítica, una de ellas la necesidad de información. El presente estudio establece los elementos que la enfermera debe tener en cuenta para abordar efectivamente la necesidad de información. Método: revisión integrativa de la producción científica generada entre 2007 y 2016. Se incluyeron 23 artículos, por su aporte en la comprensión de los elementos para abordar la necesidad. Resultados: se establecieron seis categorías que denotan los elementos para el abordaje: características de la información, valoración e identificación de la necesidad de información, habilidades indispensables de la enfermera para abordar la necesidad de información, participación familiar en el cuidado para satisfacción de necesidad de información, condiciones para abordar la necesidad de información y tipo de información. Conclusiones: estos elementos facilitarán el abordaje de la enfermera y contribuirán así a satisfacer la necesidad de información, mejorar la calidad de vida de los familiares y los estándares de calidad en la atención, además de contribuir a la formación académica de los profesionales con elementos basados en la evidencia científica

Palabras clave: necesidad, información, familia, UCI, enfermería.

\section{Abstract:}

Introduction: Holistic care of a critical patient should include approaching her/his family, as they are living specific needs caused by this critical situation. One of them is the information need. This study sets the elements that a nurse should take into account to effectively approach those information needs. Methods: It is a unifying review of the scientific production on this topic between 2007 and 2016. Twenty-three articles were included due to their contribution to understand the elements required to approach the needs described herein. Results: Six categories were defined to denote the elements for the nursing approach: information characteristics; assessment and identification of the information needs; essential skills in a nurse for approaching the information needs; family participation in the care so as to satisfy their information needs; conditions to approach the information needs; and type of information. Conclusions: These elements will make easier for a nurse to approach the family and will contribute to fulfil their information needs, to improve their quality of life, and enhance the quality standards in the health care. In addition, it contributes to the educative formation of health professionals thanks to the items based on the scientific evidence.

Keywords: need, information, family, ICU, nursing.

\section{Resumo:}

Introdução: $\mathrm{O}$ cuidado do paciente crítico de forma holística deve incluir a abordagem da família, que experimenta necessidades específicas derivadas da situação crítica, uma delas a necessidade de informação. O presente estudo estabelece os elementos que a enfermeira deve levar em conta para abordar efetivamente a necessidade de informação. Método: Revisão integrativa da produção científica gerada entre 2007 e 2016. Incluíram-se 23 artigos, pela sua contribuição na compreensão dos elementos para abordar a necessidade. Resultados: Foram estabelecidas seis categorias que denotam os elementos para a abordagem: características da informação, valoração e identificação da necessidade de informação, habilidades indispensáveis da enfermeira para abordar a necessidade de informação, participação familiar no cuidado para satisfação de necessidade de informação, condições para

Notas de autor

a Autora de correspondencia. Correo electrónico: lcboadaq@unal.edu.co 
a necessidade e tipo de informação e tipo de informação. Conclusões: Estes elementos facilitarão a abordagem da enfermeira e contribuirão assim a satisfazer a necessidade de informação, melhorar a qualidade de vida dos familiares e os padrões de qualidade na atenção, além de contribuir à formação académica dos profissionais com elementos baseados na evidência científica.

Palavras-chave: necessidade, informação, família, UTI, enfermagem.

\section{Introducción}

La unidad de cuidados intensivos (UCI) es un área especializada y compleja donde las enfermeras desempeñan un papel muy importante, al desarrollar una relación terapéutica con la familia en un ambiente altamente tecnológico, cuyo reto es mantener la humanización del cuidado (1,2). La base práctica disciplinar es la interrelación enfermera-paciente-entorno (3), y dentro de este último hay un elemento muy importante la familia, quien tiene una relación positiva con la recuperación del paciente cuando es abordada adecuadamente.

La UCI ha sido considerada un "ambiente estresante tanto para el personal que allí labora como para los pacientes y sus familiares" (4); por esto, "la experiencia de la hospitalización de algún ser querido en una UCI supone una crisis vital” (5,6,7), ante la cual enfermería debe brindar un cuidado holístico y humano. En general, la literatura demuestra que en la UCI es escasa la relación enfermera-familia, por la restricción en los horarios de visita, por la sobrecarga laboral, por la falta de personal, por el aprovechamiento durante la visita para realizar actividades administrativas o de descanso, por la ausencia de preparación de los profesionales para abordar emocional y psicosocialmente la familia, por utilizar la evasión como mecanismo de defensa, que generan una actitud fría, distante e inhumana $(5,8)$.

Teniendo en cuenta la problemática anterior y siendo la necesidad de información una de las principales descritas en la literatura científica experimentadas por las familias en la UCI (9), se plantea como objetivo de la investigación: establecer los elementos para abordar la necesidad de información de las familias en la UCI mediante una revisión integrativa que permita una presentación crítica de la evidencia científica que la describe. Esto a fin de impactar positivamente la calidad de vida de los familiares y la atención asistencial del paciente, lo que concuerda con las políticas internacionales de calidad en salud (1).

El avance disciplinar que determinan los elementos para abordar la necesidad de información de las personas que tienen miembros de su familia en una UCI responde al vacío de conocimiento de un cuidado familiar no protocolizado (10), el cual garantiza una uniformidad en la atención y un mejoramiento de los estándares de calidad. Los seis elementos encontrados en torno a la necesidad de información que serán descritos a continuación son: 1) características de la información, 2) valoración e identificación de la necesidad, 3) habilidades indispensables de la enfermera para abordar la necesidad, 4) participación familiar en el cuidado para la satisfacción de la necesidad, 5) condiciones para abordar la necesidad y 6) tipo de información.

\section{Metodología}

La investigación se desarrolló por medio de una revisión integrativa en la cual, críticamente, se analizaron los datos relacionados con el fenómeno de interés después de realizar una búsqueda bibliográfica; en este caso específico sobre la necesidad de información de las familias en la UCI. Ello permitió "identificar, analizar y sintetizar los resultados de los estudios independientes con el fin de determinar cuál es el estado actual del conocimiento en un área específica" (11) y dar como producto una revisión de opiniones integradoras basadas en la evidencia que aporten a las mejores prácticas $(11,12)$.

Para garantizar el desarrollo y el rigor metodológico en la revisión integrativa fue necesario determinar las siguientes etapas: 


\section{Elaboración de la pregunta orientadora de la revisión}

¿Cuáles son los elementos para abordar la necesidad de información de las familias en la UCI que la enfermera debe tener en cuenta? Teniendo claro el alcance del objetivo general: establecer los elementos para abordar la necesidad de información de las familias en la UCI a través de una revisión integrativa que permita una presentación crítica de la evidencia científica $(13,14,15,16,17)$.

\section{Búsqueda de literatura}

Se adelantó una búsqueda de artículos en las bases de datos ScienceDirect, Biblioteca Virtual en Salud (BVS), Elsevier, SciELO, Pudmed, FreeFull PDF, Embase, Redalyc, Google Académico, Medline y Scopus, bajo los descriptores necesidad AND información AND familia AND UCI AND enfermería. Los criterios de selección para los artículos científicos se muestran en la tabla $1(13,14,15,16,17)$.

TABLA 1.

Criterios de selección

\begin{tabular}{ll}
\hline \multicolumn{1}{c}{ Criterios de inclusión } & \multicolumn{1}{c}{ Criterios de exclusión } \\
\hline $\begin{array}{l}\text { Artículos originales de diferentes niveles de } \\
\text { evidencia, principalmente de enfermería. }\end{array}$ & Artículos no originales: editoriales, reflexiones. \\
\hline $\begin{array}{l}\text { Publicaciones de los últimos } 10 \text { años en revistas } \\
\text { indexadas (2007-2016). }\end{array}$ & \begin{tabular}{l} 
Publicaciones realizadas antes del 2007. \\
\hline $\begin{array}{l}\text { Artículos escritos en español, inglés y } \\
\text { portugués. }\end{array}$
\end{tabular} \\
\hline $\begin{array}{l}\text { Artículos científicos desarollados con familias científicos desarrollados con familias } \\
\text { de personas adultas en la UCI. }\end{array}$ & \\
\hline
\end{tabular}

Fuente: elaboración propia

Se identificaron 210 referencias asociadas con la temática. El límite de la búsqueda fue la saturación de datos (18). La figura 1 es el diagrama de flujo de la estrategia de búsqueda de la información.

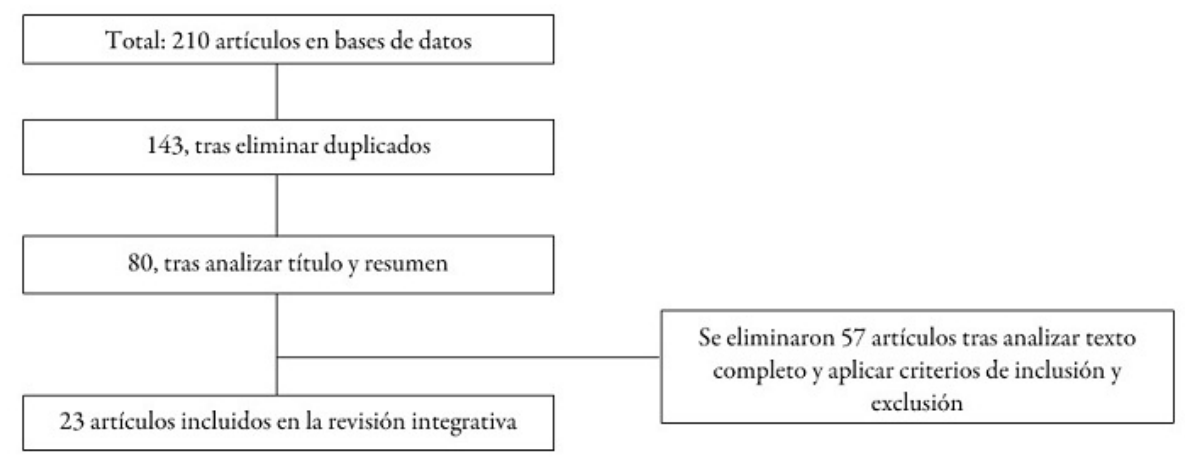

FIGURA 1.

Diagrama de flujo de los artículos incluidos y excluidos

Fuente: elaboración propia

\section{Evaluación y análisis de los datos}

Estas etapas se desarrollaron de forma simultánea, y para evaluar la calidad metodológica y determinar los datos relevantes de los artículos científicos se utilizó como instrumento el análisis o lectura crítica basada en la subestructuración, a través del cual se estableció la validez interna (metodología y riesgo de sesgos) y la validez externa (reproducibilidad de los resultados) de los artículos. Se elaboró una matriz con las fichas de análisis que tenían en cuenta los siguientes aspectos: código, base de datos, revista, año/autores, título, objetivo, 
diseño, enfoque conceptual, resultados, conclusiones y nivel de evidencia de los artículos científicos (reducción y visualización de datos $)(14,15,16,17)$.

Los datos se compararon teniendo en cuenta la identificación de temas comunes y afines a través de un patrón repetitivo extraído de la información consolidada en el análisis. Esta agrupación sistemática permitió finalmente la extracción de conclusiones y la verificación, basado en el análisis interpretativo de la descripción de los patrones comunes del fenómeno de estudio, a partir de lo cual se originan las categorías de los resultados $(14,15,16,17,18,19)$.

\section{Resultados}

En la presente revisión integrativa, los artículos científicos se clasificaron por niveles de evidencia (tabla 2). De esta manera, se encontró que la gran mayoría está en los niveles descriptivos 5 (12 artículos) y 6 (6 artículos), lo cual permite inferir que se ha definido ampliamente la necesidad de información para las familias con miembros en la UCI. Sin embargo, aún no se han desarrollado suficientes trabajos investigativos experimentales que apliquen intervenciones efectivas para satisfacer la necesidad, de tal manera que la línea de conocimiento en el fenómeno debe enfocarse en este tipo de investigaciones aplicadas. El desarrollo de esta revisión integrativa establece los elementos para el abordaje de la necesidad de información de las familias en la UCI, porque se determinan las pautas de la intervención y se da el primer paso para, posteriormente, en otras investigaciones ser llevadas a la práctica. Ello contribuye al avance del conocimiento del fenómeno y, por ende, a la solución de la problemática, al facilitar a la enfermera su quehacer, retomando en sus protocolos de atención una base científica al momento de brindar la información, y al garantizar un proceso completo y adecuado que contribuya a la satisfacción de la necesidad de información de la familia del paciente en situación crítica.

TABLA 2.

Clasificación de niveles de evidencia de los artículos incluidos en la revisión integrativa

\begin{tabular}{llr}
\hline $\begin{array}{l}\text { Nivel de } \\
\text { evidencia }\end{array}$ & Tipo de estudio & $\begin{array}{r}\text { Número de } \\
\text { artículos }\end{array}$ \\
\hline 1 & Revisión sistemática, metanálisis & 1 \\
\hline 2 & Ensayo clínico aleatorizado & 1 \\
\hline 3 & Estudios de cohorte prospectivos & 2 \\
\hline 4 & Estudios de casos y controles & 1 \\
\hline 5 & Estudios transversales & 12 \\
\hline 6 & $\begin{array}{l}\text { Estudios cualitativos con análisis a } \\
\text { profundidad }\end{array}$ & 6 \\
\hline 7 & Opinión de expertos & 0 \\
\hline
\end{tabular}

Fuente: adaptado de C. Galvao (19)

El análisis de los artículos seleccionados mostró que existen elementos que se consideran de suma importancia para satisfacer la necesidad de información de la familia del paciente en la UCI, los cuales se agruparon en seis categorías: características de la información, valoración e identificación de la necesidad de información, habilidades indispensables de la enfermera para abordar la necesidad, participación familiar en el cuidado para satisfacción de la necesidad, condiciones para abordar la necesidad y tipo de información, que se describen en la discusión.

\section{Discusión}

La hospitalización del paciente en la UCI es una situación inesperada que genera estrés en el paciente y su núcleo familiar. Ello origina necesidades familiares: emocionales, cognitivas, sociales y prácticas, dentro de las 
cuales se destaca como principal la necesidad de información (cognitiva), que hace referencia al conocimiento de la situación actual del paciente. En ella se deben tener en cuenta diferentes aspectos que se describen a continuación, de tal forma que se aborde esta necesidad de manera adecuada y permita su satisfacción, a la vez que se minimiza la carga emocional negativa que desencadene un estrés postraumático en la familia $(9,20,21)$.

\section{Características de la información}

El análisis de la evidencia científica muestra diferentes autores que describen características, cualidades o atributos que debe tener la información brindada a los familiares en la UCI. Algarbe y Vílchez mencionan que las características son: completa, clara, oportuna, precisa y exacta, por lo que se convierten en el pilar para el afrontamiento familiar de la situación crítica (22). Por otro lado, Buckley y Andrews (23) afirman que los familiares que reciben una información veraz disminuyen el estrés de la hospitalización en la UCI. En ocasiones, los enfermeros se ven implícitos a innovar ante un cambio en la condición clínica que amerite un nuevo direccionamiento de la información.

Del cumplimiento de estas características y otras que se encuentran resumidas en la tabla 3 depende en gran medida la adaptación, que evita la carga emocional negativa y, por ende, el estrés postraumático, haciendo más comprensible la situación crítica para el entorno inmediato del paciente, la familia $(10,24,25)$.

TABLA 3.

Características de la información

- Completa, clara, oportuna, precisa, exacta y veraz $(21,22,23,26,27)$.

- Innovación en el momento de informar cambios en la situación clínica $(21,23,26,27)$.

- Claridad: usar lenguaje sencillo y comprensible (28).

- Progresiva y personalizada: de acuerdo con la dinámica y evolución del paciente (29).

- Fehaciente, contrastable y suficiente (27,28).

- Adecuada y honesta. Mostrar interés por el paciente (30-32).

- Organizada, comprensible, secuencial y sistemática (33).

- Real y objetiva: no generar falsas expectativas (33). Evitar ser alarmista y conscientemente tendenciosa o parcializada (27).

Fuente: elaboración propia

\section{Valoración e identificación de la necesidad de información}

Es necesario valorar las necesidades de la familia para, posteriormente, intervenirlas y, de esta forma, hacer la experiencia de la situación crítica más comprensible, al evitar la carga emocional negativa y el estrés postraumático $(23,30,34,35,36,37)$. En general, los autores denotan al Critical Care Family Needs Inventory (CCFNI) como un instrumento clave para identificar las necesidades de las familias en la UCI $(20,23,32,38,39,40)$.

Algarbe y Vílchez (22) respaldan la familia como sujeto de cuidado en la UCI, al igual que McKiernan y McCarthy (41), haciendo referencia al holismo de enfermería dentro del cual debe reconocerse y satisfacer las necesidades de la familia del paciente. Conocer las demandas reales de la familia es la base para determinar las actividades de cuidado.

Según McKiernan y McCarthy (41), en la valoración de las necesidades de la familia, se deben tener en cuenta factores como: enfermedades inesperadas, lesiones, deterioro de la condición del paciente, dolor, sufrimiento e instrumentos tecnológicos. Es importante considerar que el primer contacto con la UCI genera expectativa, impresión y asombro, haciendo evidente la necesidad de información (39). El contacto 24 horas de enfermería favorece la identificación de las necesidades para posteriormente intervenirlas $(23,42)$.

Dentro de la valoración, el lenguaje verbal y no verbal de la enfermera establece el grado de empatía con los familiares, y ello facilita la expresión de sus necesidades (34). Así mismo, otros autores mencionan que la valoración de la necesidad debe tener en cuenta las experiencias previas, así como el nivel cultural y educativo 
de los familiares, para identificar la forma apropiada (lenguaje, metodología) de dar la información, a fin de garantizar que se entiende $(23,27,38)$.

\section{Habilidades indispensables de la enfermera para abordar la necesidad de información}

Buckley, apoyado por Algarbe y Vílchez (22) y García et al. (28) mencionan que la habilidad clínica permite a la enfermera actuar con precisión, rapidez y decisión, manteniendo la calma ante situaciones críticas, transmitiendo seguridad y confianza al paciente y su familia (23). Complementando esto, McKiernan y McCarthy (41) refieren que las primeras seis horas posteriores al ingreso se centran en la atención del paciente para lograr la estabilidad fisiológica; de ahí el importante papel de esta habilidad. Adicionalmente, se da la acogida familiar $(43,44,45,46)$.

Los autores mencionan que el éxito de la atención de enfermería depende de una adecuada formación académica, ética y humana $(23,28,29,44)$. En resumen, las habilidades que el enfermero debe tener para abordar la necesidad de información son: experticia clínica, liderazgo y facilidad para establecer relaciones interpersonales (comunicación, interacción y empatía) $(22,23,26,28,29,32,33,34,40,41,42,43,44,45,46,47)$.

\section{Participación familiar en el cuidado para satisfacción de la necesidad de información}

Definida según Algarbe y Vílchez (22) como "el proceso por el que uno o varios miembros significativos de la familia se involucran de forma voluntaria, gradual y guiada, en el cuidado del paciente". Según la anterior definición, la información está inmersa en ella al ser un proceso guiado. Para alcanzar la participación familiar en el cuidado, necesariamente, debe haber un proceso informativo continuo que permita suplir la necesidad, lo que contribuye a la adaptación familiar, a la toma de decisiones y, a su vez, a la recuperación del paciente $(42,47,48)$.

La literatura científica evidencia dos instrumentos para medir la satisfacción de las necesidades familiares, entre ellas la información, pues no existe una herramienta que solamente la evalúe; los instrumentos mencionados son el cuestionario Needs Met Inventory (NMI) y el Family Satisfaction Survey (FS-ICU) $(49,50)$.

Errasti y Tricas (51) resaltan cómo mediante estrategias de visita abierta se propician mayores espacios de interacción e información entre el personal de salud y el familiar, a fin de garantizar su participación en el cuidado. En la tabla 4 se mencionan algunas formas de participación familiar, incluyendo cuidados.

TABLA 4.

Participación familiar en el cuidado para satisfacción de la necesidad de información

- Actividades y cuidados que la enfermera puede delegar con supervisión: alimentación, aseo bucal, cambios de posición, lubricación de piel y labios, ejercicios de prevención de desacondicionamiento físico (29).

- Otras formas de participación familiar incluyen: actuación como un sustituto que toma decisiones, ser un donante de cuidado (cuidados básicos), abogar por los deseos del paciente, evaluar el sufrimiento del paciente, comunicación de información entre paciente y personal de salud, y ser una fuente de esperanza y consuelo (47).

Fuente: elaboración propia

\section{Condiciones para abordar la necesidad de información}

La literatura sobre el tema denota la comodidad, la privacidad, el confort y la empatía, las cuales no solo garantizan la satisfacción del paciente y la familia, sino la del mismo profesional en torno a la calidad de la atención prestada $(22,28,33,42,52)$. 
García et al. (28) y Llamas et al. (29) mencionan como elemento importante el flexibilizar los horarios de visita de acuerdo con las necesidades y responsabilidades de la familia, informar cambios imprevistos en la evolución, preservar la intimidad y permitir disponer de objetos considerados sagrados (estampas, rosarios, fotos, etc.), respetando la cultura familiar. García et al. (28) también refieren que la familia siente mayor comodidad al entablar contacto con los mismos profesionales diariamente, hecho que debe ser tenido en cuenta para la asignación de los pacientes.

Por otra parte, Gaeeni et al. (26) mencionan que "la necesidad de información es universal y extremadamente importante para todos los miembros sin importar la edad, sexo, estado socioeconómico y nivel educativo". Complementando esto, Galvis y Salamanca (39) refieren que cada experiencia de hospitalización en la UCI es diferente, individual y única; por ende, la necesidad de información siempre está presente y es similar para todos los miembros de la familia o situaciones como lo mencionan estos autores, condiciones que deben ser tenidas en cuenta por la enfermera.

\section{Tipo de información}

Especifica todo lo que la familia de los pacientes críticos debe saber y cuáles son los tipos de información que la enfermera debe brindar en cuidados intensivos (tabla 5). El conocimiento de esto, junto a los elementos brindados por las otras categorías, garantiza el éxito de la enfermera al intervenir la necesidad de información de las familias en la UCI, al dar las herramientas necesarias que guíen el proceso de información.

TABLA 5.

Tipo de información

\begin{tabular}{ll}
\hline $\begin{array}{l}\text { Información inicial o de } \\
\text { ingreso }\end{array}$ & $\begin{array}{l}\text { Situación fisica (estado clínico, monitorización, ventilación mecánica, etc.) y emocional del paciente, acceso } \\
\text { a la unidad, uso de la sala de espera, sistemas y horarios de visitas y de información. Esta información puede } \\
\text { ser oral y escrita (27,33,42). }\end{array}$ \\
\hline $\begin{array}{l}\text { Información de } \\
\text { evolución }\end{array}$ & $\begin{array}{l}\text { Estructurada según necesidades básicas: nutrición (vía oral, nutrición parenteral, visita y ayuda en comidas), } \\
\text { eliminación (espontánea, sonda vesical), termorregulación (ficbre, afebril), oxigenación (patrón } \\
\text { respiratorio, ventilación mecánica, soportes de oxígeno), actividad y reposos (movilidad, tolerancia a la } \\
\text { actividad, sueño), seguridad física (dolor, lesiones en piel y heridas quirúrgicas, aislamiento, inmovilización), } \\
\text { seguridad psíquica (orientación, estado de conciencia, estado de ánimo, sedación, relajación) (27,33,42). }\end{array}$ \\
\hline Información a demanda a da detición del familiar (resolver dudas sobre la información médica, explicaciones sobre el entorno \\
$\begin{array}{l}\text { Se da a petición } \\
\text { tecnológico y evolución en general) (27,33,42). }\end{array}$ \\
\hline $\begin{array}{l}\text { Información en } \\
\text { situaciones especiales }\end{array}$ & $\begin{array}{l}\text { Traslados fuera de la unidad, situaciones de desorientación temporo-espacial, cambios en la imagen del } \\
\text { paciente (27,33,42). }\end{array}$ \\
\hline Información al alta & Previo al traslado a piso, cuidados específicos (27,33,42). \\
\hline
\end{tabular}

Fuente: elaboración propia

Según Abianza et al. (27) y García et al. (28), para empezar a informar la enfermera debe identificar qué fase de la situación crítica atraviesa la familia:

Confusión inicial: se vive estrés e incertidumbre, no saben dónde están y a quién preguntar.

Búsqueda de información: ayuda a salir del estado de confusión inicial.

Observación del entorno: seguimiento de la calidad del cuidado que recibe el paciente.

Obtención de recursos: apoyo emocional, actividades recreativas, descanso o privacidad.

De estas fases depende la información que se le proporcione al familiar. Estos autores clasifican la información en la UCI en cuidados básicos, actitud ante el enfermo, diagnóstico principal, planes a seguir y gravedad $(7,27,28)$.

Por otra parte, diferentes autores refieren que las familias esperan que las enfermeras informen sobre los signos vitales, el cuidado, el confort y el descanso del paciente, así como sobre el tratamiento y los aspectos concretos de las UCI, como equipo tecnológico, alarmas, sistemas de monitorización, número de teléfono, equipo de profesionales que trabajan en la unidad, reglas y normas de la UCI, ubicación, sala de espera, horario de información, horario de visitas y su limitación. Además de los cuidados propios del paciente: higiene, comunicación, nutrición, ayuda espiritual, tratamiento y pronóstico $(9,28,29,43,44,47)$. Teniendo en cuenta los autores, se plantea el siguiente tipo de información de acuerdo con el transcurso de la hospitalización en UCI. 


\section{Conclusión}

La articulación conceptual-teórico-empírica de esta investigación da como resultado una propuesta aplicable en la práctica que suscite un cambio, al brindar elementos al profesional de enfermería para abordar efectivamente la necesidad de información. Ello mejora la calidad de vida de los familiares y los estándares de calidad en la atención y está en concordancia con las políticas internacionales de calidad en salud. Este avance disciplinar es grande, teniendo en cuenta que el cuidado familiar no está protocolizado y depende de la seguridad y habilidad de cada enfermera. Así es como la revisión integrativa genera resultados que también contribuyen a la formación académica de los profesionales con elementos basados en la evidencia científica.

El eje central de los resultados de la investigación es el tipo de información, al ser lo que la familia recibe tangiblemente; sin embargo, el proceso de información requiere todas las otras categorías para unas condiciones óptimas que garanticen una información efectiva que supla la necesidad.

\section{Referencias}

1. Joint Commission International. Estándares de acreditación para hospitales de Joint Commission International [Internet]. 5a ed. Oakbrook Terrace, Il; 2014. Disponible en: https://www.jcrinc.com/assets/1/14/EBJCIH1 4S_Sample_Pages.pdf

2. Resolución 2003 de 2014/28 de mayo, por la cual se definen los procedimientos y condiciones de inscripción de los prestadores de servicios de salud y de habilitación de servicios de salud [Internet]. Ministerio de Salud y Protección Social. Disponible en: https://www.minsalud.gov.co/Normatividad_Nuevo/Resoluci\%C3\%B3n\% 202003\%20de\%202014.pdf

3. Newman M, Smith M, Dexheimer M, Jones D. El núcleo de la disciplina: Una revisión. Adv Nurs Sci. 2008;31(1):E16-27.

4. Mejías M. Interacción del personal de enfermería con los familiares del paciente politraumatizado y su relación con el nivel de estrés de los familiares. Portales Médicos [Internet]. 2010 jul 15. Disponible en: https://www.salama ndra.edu.co/fileadmin/documentos/articulos_academicos/Enfemeria.pdf

5. Cerón YM. Aspectos presentes en la comunicación de la enfermera, la familia y la institución en la unidad de cuidado intensivo [trabajo de grado]. Bogotá: Universidad Pontificia Javeriana, Facultad de Enfermería; 2008.

6. Brevis I, Silva P. Creencias, sentimientos e incertidumbre frente a la enfermedad en familiares de pacientes ingresados a UCI. Rev Chil Med Intensiva. 2011;26(1):27-34.

7. Parra M, Guáqueta S, Triana M. Perspectivas del cuidado de enfermería en UCI: Una visión desde el modelo de cuidado HANC. Bogotá: Kimpres; 2012.

8. Vásquez M, Eseverri MC. El cuidado de las familias en las unidades de cuidados intensivos desde la perspectiva de Jean Watson. Enferm Intensiva. 2010;21(4):161-4.

9. Pardavila MI, Vivar CG. Necesidades de la familia en las unidades de cuidados intensivos: Revisión de la literatura. Enferm Intensiva. 2012;23(2):51-67.

10. Campo MC, Cotrina MJ. Relación de ayuda al familiar del paciente en situación crítica. Enferm Global. 2011;24:103-9.

11. Burns N, Grove S. Revisión de la literatura científica sobre los estudios publicados. En Investigación en enfermería: Desarrollo de la práctica enfermera basada en la evidencia. 5a ed. Madrid: Elsevier; 2012. pp. 194-233.

12. Normatividad sobre derechos de autor y propiedad intelectual en Colombia [Internet]. Disponible en: https://w ww.cide.edu.co/cidevirtual/file.php/1/Normatividad_Derechos_de_Autor.pdf

13. Soares C, Komura L, Peduzzi M, Sangaleti C, Yonekura T, Audebert D. Integrative review: concepts and methods used in nursing. Rev Esc Enferm USP. 2014;48(2):329-39. Disponible en: https://www.ee.usp.br/reeusp/

14. Tavares M, Dias M, Carvalho R. Integrative review: what is it? How to do it? Einstein. 2010; 8(1):102-6. 
15. Whittemore R, Knafl K. Methodological issues in nursing research. The integrative review: updated methodology. J Adv Nurs. 2005;52(5):546-53.

16. Manterola C, Zavando D. Cómo interpretar los "Niveles de Evidencia" en los diferentes escenarios clínicos. Rev Chil Cir. 2009;61(6):582-595.

17. Manterola C, Asenjo-Lobos C, Otzen T. Jerarquización de la evidencia: Niveles de evidencia y grados de recomendación de uso actual. Rev Chil Infectol. 2014;31(6):705-718.

18. Crossetti, MGO. Revisão integrativa de pesquisa na enfermagem o rigor cientifico que lhe é exigido [editorial]. Rev Gaúcha Enferm. 2012;33(2):10-1.

19. Galvao C. Niveles de evidencia [Editorial]. Acta Paul Enferm. 2006;19(2):7.

20. Domingo M, García A, López I, Benito R, Peláez R, Rojas A. Detección de necesidades de los familiares de pacientes ingresados en la Unidad de Ictus. Rev Cient Soc Esp Enferm Neurol. 2012;35(1):6-11.

21. González DS, Álvarez DM, Sánchez CP. Inventario de necesidades de los familiares de los pacientes egresados de la unidad de cuidado intensivo. Rev Cien Cuidad. 2014;11(2):46-58.

22. Algarbe $S$, Vílchez V. Necesidades de las familias de pacientes internados en la unidad de terapia intensiva de adultos. Notas de Enfermería. 2011;11(17):5-10.

23. Buckley P, Andrews T. Intensive care nurses' knowledge of critical care family needs. Intensive Crit Care Nurs. 2011;27:263-72.

24. Yépez Y. Comunicación que establece el profesional de enfermería con los familiares de los pacientes hospitalizados en la unidad de cuidados intensivos del hospital "Dr. José Gregorio Hernández" adscrito a la alcaldía metropolitana, Caracas. Durante el segundo semestre 2009 [Trabajo de grado]. Caracas: Universidad Central de Venezuela, Facultad de Medicina, Escuela de Enfermería; 2009.

25. García D, Estrada M, Gallegos M, Antuna A. Efectividad del modelo de cuidado de enfermería para la familia de paciente con infarto agudo al miocardio en la unidad de cuidados intensivos. Cuidarte. 2015;6(1):923-31.

26. Gaeeni M, Farahani M, Seyedfatemi N, Mohammadi N. Informational support to family members of intensive care unit patients: the perspectives of families and nurses. Global J Health Sci. 2015;(7)2:8-19. https://dx.doi. org/10.5539/gjhs.v7n2p8

27. Abizanda R, Bernat A, Ballester R, Bisbal E, Vidal B, Cubedo M, et al. Estrategias de información en una unidad de cuidados intensivos polivalente. Med Intensiva. 2008;32(5):216-21.

28. García A, Sánchez F, Amorós SM, Balaguer F, Diéz M, Durán MT, et al. Desarrollo de una guía de atención a los familiares del paciente crítico. Enferm Intensiva. 2010;21(1):20-7.

29. Llamas F, Flores J, Acosta ME, González J, Albar MJ, Macías C. Necesidades de los familiares en una unidad de cuidados críticos. Enferm Intensiva. 2009;20(2):50-7.

30. Gómez P, Soriano JF, Monsalve V, Ibáñez A. Satisfacción con la información: posible variable interviniente en el estado de ánimo de cuidadores primarios de pacientes críticos. Clínica y Salud. 2009;20(1):91-105.

31. Vargas JE, Aguilar EL. Niveles de estrés en los familiares de los pacientes atendidos en la unidad de cuidados intensivos de un hospital general. Centr Regi Investig Psicol [Internet]. 2011;5(11):35-40. Disponible en: https://www.conductitlan.net/centro_regional_investigacion_psicologia/60_estres_familiares_cuidados_i ntensivos.pdf

32. Hashim F, Hussin R. Family needs of patient admitted to intensive care unit in a public hospital. Procedia-Social and Behavioral Sciences. 2012;36:103-11.

33. Pérez MC, Najarro FC, Dulce MA, Gallardo N, Fernández A. Comunicación: Una necesidad para el pacientefamilia. Una competencia de enfermería. Revista Digital de Enfermería [Internet]. 2009;1(3):15-20. Disponible en: https://www.paginasenferurg.com/revistas/2009/septiembre/comunicacion.pdf

34. Blanca JJ, Blanco AM, Luque M, Ramírez MA. Experiencias, percepciones y necesidades en la UCI: Revisión sistemática de estudios cualitativos. Enferm Global. 2008;12:1-14.

35. Luque M. Fundamentos teóricos de la familia como parte del paciente. Portales Médicos [Internet]. 2013 feb 4. Disponible en: https://www.revista-portalesmedicos.com/revista-medica/familia-paciente/ 
36. Bernal D, Horta S. Cuidado de enfermería para la familia del paciente crítico desde la teoría de la comprensión facilitada: Artículo de innovación para la práctica. Enferm Univ. 2014;11(4):154-63.

37. Ballesteros G. Comunicación entre la enfermera y el familiar de la persona hospitalizada en la unidad de cuidados intensivos [Tesis de maestría]. Bogotá: Universidad Nacional de Colombia; 2014.

38. Santana K, Kimura M, Azevedo K. Necesidades de los familiares de pacientes en unidades de terapia intensiva: Análisis comparativo entre hospital público y privado. Rev Latino-am Enfermagem. 2007;15(1).

39. Galvis CR, Salamanca E. Percepción de necesidades en cui $\neg$ dadores familiares de adultos internados en una unidad de cuidados intensivos de una institución prestadora de salud (IPS) privada en Villavicencio, Colombia. Investig En $\neg$ ferm Imagen Desarr. 2014;16(2):81-94. https://doi.org/10.11144/Javeriana.IE16-2.pncf

40. López MA, Pérez M, Merino E, Taurá G, Quispe LC, Manzanedo D, et al. Efecto de un protocolo de acogida familiar en una unidad de cuidados sobre la encuesta de satisfacción. Nursing. 2011;29(8):55-65.

41. McKiernan M, McCarthy G. Family members' lived experience in the intensive care unit. Intensive Crit Care Nurs. 2010;26:254-61.

42. Alfonso DP, Fernández M, García S, Contreras I, Cumbreras EM, Martín B. Información enfermera: Satisfacción de los familiares de pacientes en cuidados críticos. Biblioteca Lascasas [Internet]. 2007;3(1). Disponible en: htt ps://www.index-f.com/lascasas/documentos/lc0215.php

43. Gallardo N, Najarro FR, Dulce MA, Pérez MC, Fernández A. Calidad en la atención e información: Opinión de los familiares. Revista Digital de Enfermería. 2009;1(3):21-4.

44. Santana L, Sánchez M, Hernández E, García M, Eugenio P, Villanueva A. Necesidades de los familiares de pacientes de cuidados intensivos: percepción de los familiares y del profesional. Med Intensiva. 2007;31(6):273-80.

45. Aguilar R. La comunicación en enfermería: El canal hacia la satisfacción profesional. Estudio comparativo entre dos hospitales [Tesis de maestría]. Barcelona: Escuela Universitaria de Enfermería Santa Madrona; 2004.

46. Ramón R, Segura M, Palanca M, Román P. Habilidades sociales en enfermería: El papel de la comunicación centrada en el familiar. Rev Esp Comun Salud. 2012;3(1):49-61.

47. Wilson M, Kaur S, Gallo A, Pickering B, Gajic O, Herasevich V. Important clinician information needs about family members in the intensive care unit. J Crit Care. 2015;30:1317-23.

48. Márquez M, Carrillo GM. La experiencia del familiar de la unidad de cuidados intensivos en Bucaramanga (Colombia): Un estudio fenomenológico. Arch Med. 2015;15(1):95-106.

49. Olano M, Vivar C. Instrumentos para la valoración de las necesidades de los familiares de pacientes ingresados en cuidados intensivos: una revisión sistemática. An Sist Sanit Navar. 2012;35(1):53-67. https://dx.doi.org/10.43 21/S1137-66272012000100006

50. Lam SM, So HM, Fok SK, Li SC, Ng CP, Lui WK, et al. Intensive care unit family satisfaction survey. Hong Kong Med J. 2015;21(5):435-43. https://doi.org/10.12809/hkmj144385

51. Errasti B, Tricas S. La visita flexible en las unidades de cuidados intensivos: beneficios para los familiares del paciente crítico. Enferm Intensiva. 2012;23(4):179-88. https://dx.doi.org/10.1016/j.enfi.2012.08.001

52. Goñi I. Algunas reflexiones sobre el concepto de información y sus implicaciones para el desarrollo de las ciencias de la información. ACIMED. 2000;8(3):201-7.

\section{Notas}

* Artículo de revisión integrativa

1. Financiamiento: ninguno

2. Conflictos de interés: los autores declaran no tener conflictos de interés.

\section{Licencia Creative Commons CC BY 4.0}

Cómo citar este artículo: Boada Quijano LC, Guáqueta Parada SR. Elementos para abordar la necesidad de información de las familias en una unidad de cuidados intensivos. Investig Enferm Imagen Desarr. 2020;21(2). https://doi.org/10.11144/Javeriana.ie21-2.eani 